\title{
Distributed Geometric Distance Estimation in Ad Hoc Networks
}

\author{
Sabrina Merkel, Sanaz Mostaghim, and Hartmut Schmeck \\ Institute AIFB, Karlsruhe Institute of Technology (KIT) \\ 76128 Karlsruhe, Germany \\ \{sabrina.merkel, sanaz.mostaghim, hartmut.schmeck\}@kit.edu \\ wWW.aifb.kit.edu
}

\begin{abstract}
Distributed localization algorithms for nodes in ad hoc networks are essential for many applications. A major task when localizing nodes is to accurately estimate distances. So far, distance estimation is often based on counting the minimum number of nodes on the shortest routing path (hop count) and presuming a fixed width for one hop. This is prone to error as the length of one hop can vary significantly. In this paper, a distance estimation method is proposed, which relies on the number of shared communication neighbors and applies geometric properties to the network structure. It is shown that the geometric approach provides reliable estimates for the distance between any two adjacent nodes in a network. Experiments reveal that the estimation has less relative percentage error compared to a hop based algorithm in networks with different node distributions.
\end{abstract}

Keywords: Ad Hoc networks, localization, distance estimation.

\section{Introduction}

Mobile ad hoc networks (MANETs), a network of devices with local communication ability and without a fixed topology have been more and more subject to research. In such networks, adding a GPS-receiver to the devices might not always be desirable, for example due to power consumption or cost issues. In addition, GPS does not help in indoor or underwater scenarios. Nevertheless, location-awareness plays an important role such as for the allocation of event reporting in a monitoring sensor network [1], location dependent routing [4, 8 ] assistance of group querying [9], pattern formation [10,11] and many more. For that reason, alternative localization techniques were proposed to derive the location of each device in the network (cf. [12 14]).

Many of these algorithms use a small number of so called anchor nodes which are assumed to know their own coordinates either through a GPS-receiver or due to a priori configuration. Examples for such algorithms are given in [15-20]. Many of these algorithms rely on an estimate of the distance between each node and the anchors to calculate the nodes' coordinates. There are several methods for estimating distances in ad hoc networks. The most commonly addressed approach uses the strength of the radio frequency signal [21 24] or the time-of-flight 
analyzes of the signal [25, 26]. Both technique require suitable hardware which might not always be available. To avoid this problem, mathematical approaches have been developed, mostly counting communication hops between the node and an anchor and multiplying this value with an estimate for the width of one hop [21,27 31. Different from the existing approaches, the main idea of GeoDE is based on estimating the distance between two adjacent nodes taking into account the individual local conditions.

The idea to derive a distance estimate from the number of shared communication partners was first presented in [32, and later on refined in [33, 34]. In [33] the ratio of shared to total communication partners was used for the first time and the mapping between this ratio and the distance of two adjacent nodes was derived through empirical studies. In 34] a first order Taylor series expansion is applied to approximate the mapping function. Here, an alternative approach to the approximation in 34] is proposed. Furthermore, a technique of averaging estimation results between neighbors is introduced and it is shown that this improves the robustness in non-uniformly, distributed networks. Additionally, an algorithm is presented to derive long range distance estimation from the estimates between adjacent nodes which can be used to estimate distances to remote anchor nodes for subsequent localization, for example using multilateration [15]. Experiments are conducted to analyze the performance of GeoDE and the influence of identified error sources. GeoDE is examined in two scenarios (a) computing the distance between any two neighbors in the network and (b) computing the distance between all nodes and one anchor node. The behavior of GeoDE is tested in different network scenarios and for varying signal radius of the devices. The results indicate that the estimation using GeoDE is more accurate than estimates derived by a hop based algorithm.

The applied model of an ad hoc network assumes randomly distributed devices on a two dimensional obstacle free plane. The devices do not have global knowledge of the network topology or their locations. Each device can communicate with adjacent devices, i.e. all devices in its neighborhood. The neighborhood of a device is defined as a physical neighborhood on the plane within a fixed distance $r$ from the device. The radius $r$ is identical and known to all devices and assumed to be much smaller than the dimensions of the plane. All devices are assumed to have the same properties (homogeneous devices), except for anchor devices which posses knowledge of their own positions. Even though mobility is not regarded in this paper, the adjustment of the presented distance estimation algorithm to a mobile network is straightforward.

This paper is structured as follows. In Section 2, the GeoDE algorithm is specified. Section 3 presents the experiments' settings and displays and discusses the results. Section 4 concludes the paper.

\section{Distributed Geometric Distance Estimation (GeoDE)}

The basic idea of GeoDE is to approximately determine the common surface of two overlapping communication areas by the ratio of shared to total neighbors. 


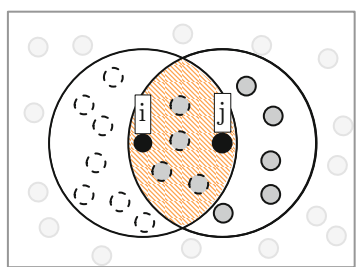

(a)

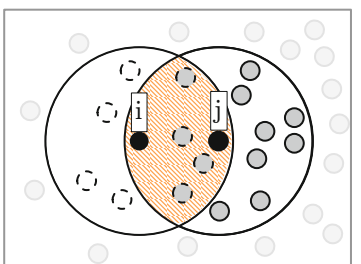

(b)

Fig. 1. Two examples for adjacent nodes $i, j$ and their neighborhoods. Nodes with dotted lines belong to $N_{i}$, Grey filled nodes to $N_{j} . S_{i j}$ are nodes in the shaded area.

Knowing the overlapping surface $O$, the distance between the two communicating nodes can be derived. The distance can then be used as input for the localization algorithms presented in Section 1 to obtain coordinates for each device. In this section, it is shown how to estimate the overlapping surface of the communication area of two adjacent nodes and the necessary steps to derive an estimate for the distance between the two nodes. The requirements for the GeoDE algorithm are that each node knows all its neighbors and can communicate with them. For node $i$ to derive the distance to its neighbor $j$ applying GeoDE, the neighbors of node $i$ have to be distinguished with respect to $j$ as follows:

Definition 1 (Classification of Neighbors). Let $i, j$ be two adjacent nodes and $N_{i}, N_{j}$ the sets of nodes situated in the neighborhood of $i$ and $j$ respectively. The neighbors of $i$ can be categorized with respect to $j$ as:

$$
\begin{gathered}
\text { shared neighbors: } S_{i j}:=\left(N_{i} \cap N_{j}\right) \\
\text { individual neighbors: } I_{i j}=\left(N_{i} \backslash S_{i j}\right)
\end{gathered}
$$

Figure 1 shows two examples for adjacent nodes $i$ and $j$ and the corresponding classification of their neighbors.

The network structure of two adjacent nodes and their communication areas can be mapped to the geometrical shape of two overlapping circles. The problem to determine the distance between the adjacent nodes is hence transfered to computing the distance between the corresponding circles' centers. The ratio of shared $S_{i j}$ to total neighbors $N_{i}$ of a node $i$ might deliver a good estimate for the ratio of overlapping to total circular surface area. Assuming this correlation holds, the surface of the overlapping area $O$ can be estimated from the perspective of node $i$ as $O \approx \pi r^{2} \cdot \frac{\left|S_{i j}\right|}{\left|N_{i}\right|}$.

The circles' cut surface $O$ has the shape of a concave lens or a mirrored circular segment with surface $A$ (cf. Figure 2), with:

$$
A \approx 0.5 \cdot \pi r^{2} \cdot \frac{\left|S_{i j}\right|}{\left|N_{i}\right|}
$$

When two circles of the same surface overlap, the cut's surface $O$ should be inverse proportional to the distance $d$ between the circles' centers. The segment 


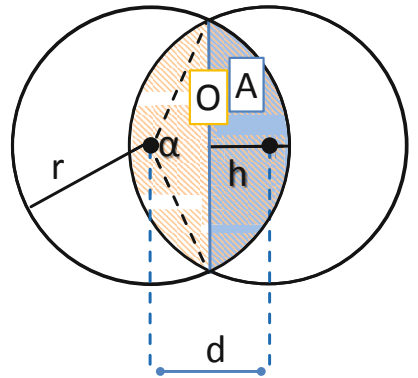

Fig. 2. Geometric characteristics of two overlapping circles

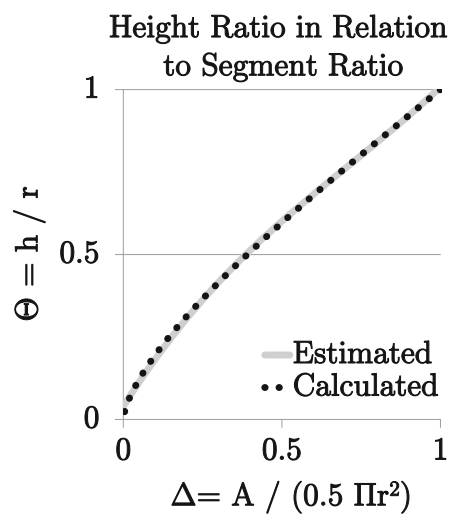

Fig. 3. Relation of $\theta$ to $\Delta$ and the approximated third-degree polynomial function $f$

surface $A$ can be calculated from a known radius $r$ and a segment height $h$ using the standard equation (2):

$$
A=r^{2} \arccos \left(1-\frac{h}{r}\right)-\sqrt{2 r h-h^{2}}(r-h)
$$

With known $A$ and $r$ one could try to derive the value of $h$ from equation (2). The segment height $h$ can be mapped to the distance $d$ between the circles' centers with known $r$. The distance between the center of the circle and the chord is equal to $r-h$. Therefore, the distance between the two centers can be obtained by:

$$
d=2 \cdot(r-h)
$$

Resolving Equation (2) to $h$ is not feasible. In [34] the first order Taylor series expansion is used to approximate equation 2 but with the following considerations an alternative solution is possible. As Equation (2) depends on $h$ and $r$ there is no 2-dimensional representation that could be approximated by using regression. Nevertheless, the following considerations help to solve this problem. The height $h$ of a segment can be described as a ratio $\theta$ of the circle's radius $r$ and the segment area $A$ is a portion of half the circle's surface:

$$
\theta=\frac{h}{r} \quad(4) \quad \Delta=\frac{A}{0.5 \cdot \pi r^{2}}
$$

In the following we show that $\Delta$ and $\theta$ are independent of $r$ with the result that the relationship between $\Delta$ and $\theta$ can be approximated using regression.

The standard equations (7) and (6) describe $A$ and $h$ depending on $r$ and angle $\alpha$ (cf. Figure 2). 


$$
A=\frac{r^{2}}{2} \cdot(\alpha-\sin (\alpha))
$$

$$
h=r \cdot\left(1-\cos \left(\frac{\alpha}{2}\right)\right)
$$

Substitution $A$ and $h$ by rearranging Equations (5) and (4), it becomes apparent that $\Delta$ and $\theta$ only depend on $\alpha$, which has a fixed value range, but are independent from $r$.

The relation of $\Delta$ and $\theta$ can be approximated using regression. Figure 3 shows data points (Grey line) and the approximated third-degree polynomial function $f: \Delta \rightarrow \theta$ (dotted line) derived through polynomial regression. Apparently, $f$ is an almost perfect approximation of the relationship between $\Delta$ and $\theta$.

From the approximated function $f$, an estimate for the segment height $h$ and, thus, the distance $d$ can be calculated with known $\Delta$ :

$$
d=2 r(1-2 \cdot f(\Delta))
$$

As stated before, $A$ can be estimated from the relation between shared neighbors $S_{i j}$ to total neighbors $N_{i}$ which can be computed locally using Equation (11).

Putting it all together, Equation (9) calculates the distance estimate $\hat{d}_{i j}$ for node $i$ to its adjacent neighbor $j$, given the number of shared neighbors $\left|S_{i j}\right|$, total neighbors $\left|N_{i}\right|$ and $r$.

$$
\left.\left.\hat{d}_{i j}=r \cdot\left(a \cdot\left(\frac{\left|S_{i j}\right|}{\left|N_{i}\right|}\right)^{3}+b \cdot\left(\frac{\left|S_{i j}\right|}{\left|N_{i}\right|}\right)^{2}+c \cdot\left(\frac{\left|S_{i j}\right|}{\left|N_{i}\right|}\right)+e\right)\right)\right)
$$

Using regression to determine the polynomial $f$ and further computations, the coefficients of the above equation can be estimated as follows:

$$
a=3.90 \quad b=-4.16 \quad c=3.04 \quad e=0.04
$$

\subsection{Evaluation of GeoDE}

The accuracy of the proposed GeoDE approach depends on two factors. Firstly, the approximation of $A$ using Equation (1) depends on the distribution of neighbors in the communication area as well as the neighborhood size $N_{i}$, secondly, the approximation of function $f$ using polynomial regression is a source of error.

The assumption underlying the GeoDE approach is that the number of nodes within an area of the environment can be mapped to the size of this area. This is a critical assumption when the distribution of nodes is imbalanced. As a result the ratio of shared to individual neighbors might not reflect the relation of overlapping to total circular area anymore. Figure 1(b) illustrates this effect. Also, the neighborhood size $N_{i}$ determines the possible precision for estimating $\Delta$. There are $\left|N_{i}\right|+1$ possible estimates for the ratio of segment surface area to total area $\Delta$. The margin between these values is $\frac{1}{\left|N_{i}\right|}$. The resulting possible absolute error for the estimation of $\Delta$ lies within the interval $\left[0, \frac{1}{\mid N_{i}}\right)$. From Equation (8) and (9) the maximum absolute distance estimation error induced 


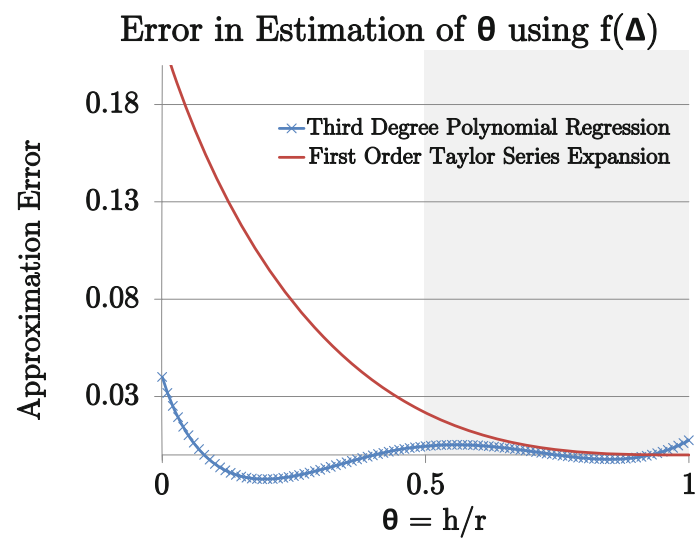

Fig. 4. The approximation error of function $f$

by a small neighborhood size can be calculated as $\epsilon \in[0,(28 a+12 b+4 c) r)$ with $\left|N_{i}\right|=1$ and $\Delta \rightarrow 1$. The impact of the nodes' distribution is assessed in the experiments shown in Section 3

The other source of error concerns the approximation of function $f$. Figure 4 shows the deviation between the approximation $f(\Delta)$ and the corresponding calculated values of $\theta$ for different values of $\theta$. Also, the approximation error using first order Taylor series expansion as suggested in 34 is printed for comparison. As Figure 4 indicates, the approximation error of function $f$ is at most of 0.04 , which leads to a maximum absolute distance estimation error of $0.16 r$. The actual error depends on the ratio of height $h$ to radius $r$ and, as the height is coupled with the distance $d$. It follows that estimating the same distance with different radii $r$ can lead to different estimation errors. Nevertheless, at least for $\theta<0.9$ the error using the polynomial approximation is smaller than using the first order Taylor series as suggested in 34 .

\subsection{Distributed GeoDE Algorithm for Ad Hoc Networks}

In principle, the distance estimate $\hat{d}_{i j}$ can range between 0 and $r$ as the centers of two overlapping circles have a maximum distance of $2 r$. This ignores the fact, that adjacent nodes can have a maximum distance of $r$ to be able to communicate. Therefore, using this concept in a network, $\hat{d}_{i j}$ can be restricted to a maximum value of $r$. This corresponds to a limited height $h \in[0.5 r, r]$ and, therefore, the approximation error of function $f$ is limited to the section highlighted in Grey in Figure 4.

As neighborhoods of $i$ and $j, N_{i}$ and $N_{j}$, commonly differ in size (cf. Figure 1 for an example), node $i$ and node $j$ calculate different estimates for the distance between them. An improved approximation can be obtained when node $i$ and node $j$ exchange their estimates via communication and calculate the average of $\hat{d}_{i j}$ and $\hat{d}_{j i}$. 
This leads to the following algorithm computed by node $i$ to estimate its distance to the adjacent node $j$ using the GeoDE approach:

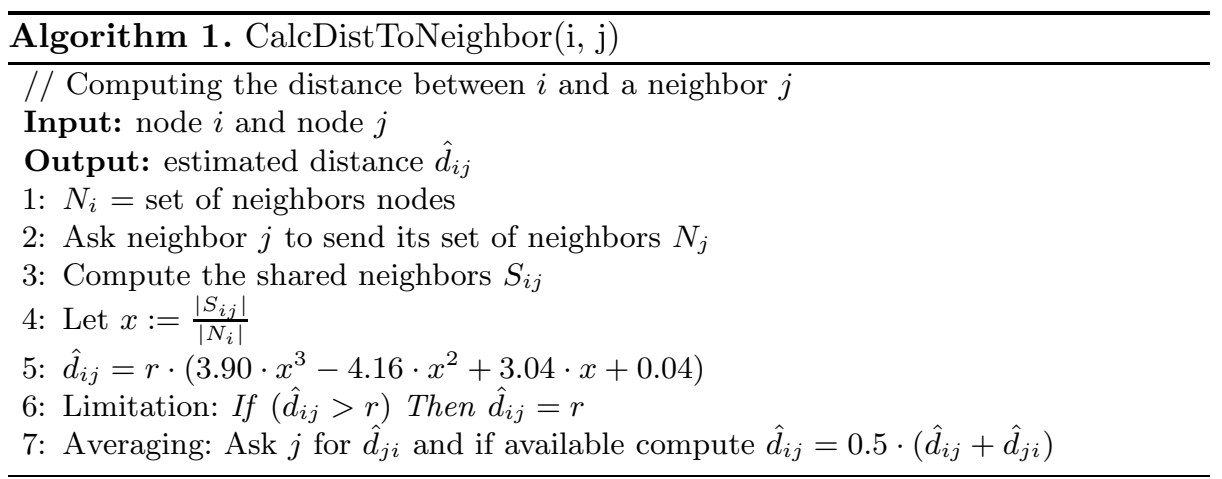

To transfer the presented concept to a long range distance estimation between a node $i$ and an anchor node $a$, all distances along the shortest path between both nodes are aggregated. The assumption is that all nodes in the network estimate their distance to the anchor node $a$, which is the case for all eligible localization algorithms (cf. Section 1). The distance between a node $i$ and an anchor $a$ can be computed using Algorithm 2.

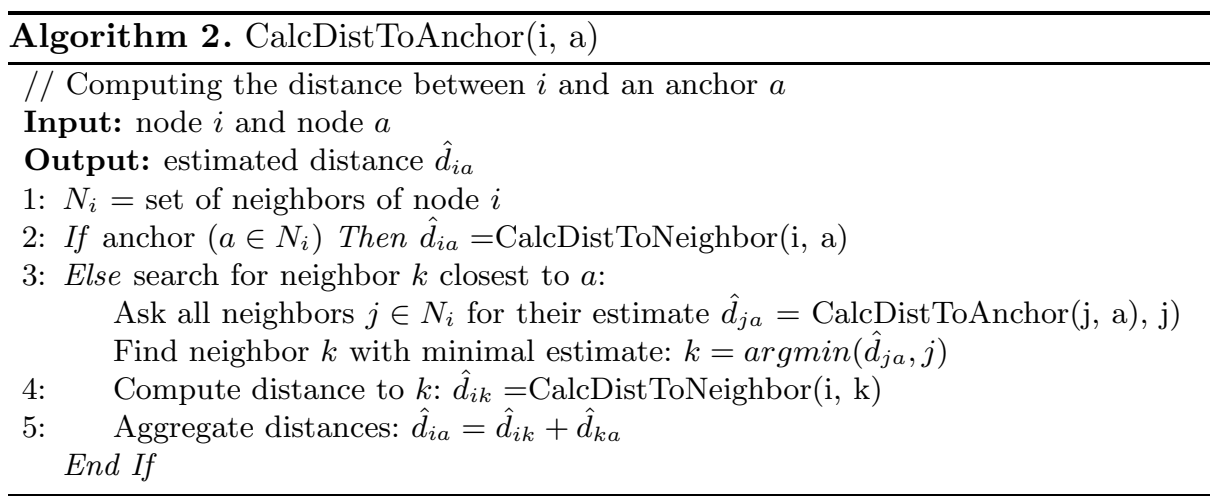

For comparison, in [15], the distance $\hat{d}_{i a}$ between a node $i$ and the anchor $a$ is estimated as:

$$
\hat{d}_{i a}=\left(\frac{\sum_{j \in N_{i}} h_{j a}+h_{i a}}{\left|N_{i}\right|+1}-0.5\right) \cdot r
$$

$h_{i a}$ denotes the hop count of node $i$ to the anchor $a$.

Note that in both algorithms each node's calculation depends on other nodes' results. Therefore, the algorithm has to be executed iteratively before a stable 


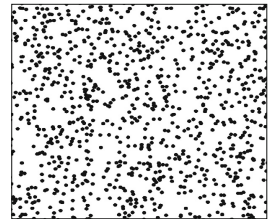

(a) Scenario 1

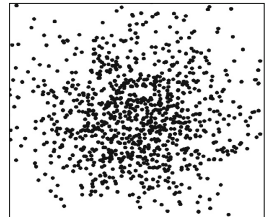

(b) Scenario 2

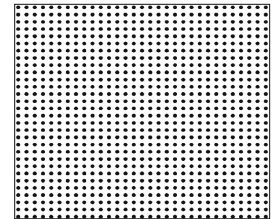

(c) Scenario 3

Fig. 5. Positioning according to a uniform random distribution (a), a Gaussian random distribution (b), and evenly distributed nodes (c)

estimate is achieved. The necessary number of executions is subject to the neighborhood size and the number of nodes that lie on the shortest path between $i$ and $a$. In mobile networks the algorithm can be executed repeatedly to dynamically compute the distance estimate considering changes in the locations of node $i$ or $a$ respectively.

\section{Experiments}

GeoDE relies on the idea that the ratio of shared to total neighbors can be used as an estimate for the ratio of overlapping to total surface of the communication area. In this section experiments are presented to evaluate whether this assumption holds for a variety of network topologies. The second part of the experiments concerns the usage of the GeoDE approach to estimate distances to anchor nodes. The results are compared with the results of the hop count based approach presented in [29].

For the experiments a 2-dimensional square environment of size $1.0 \times 1.0$ units containing 1000 nodes is considered. The neighborhood size and the distribution of nodes is expected to influence the quality of GeoDE. Therefore, three different scenarios for the nodes' distribution in the environment are considered. Two randomly distributed networks are investigated using a uniform random distribution in Scenario 1 and a Gaussian random distribution in Scenario 2. In Scenario 3, the nodes are evenly positioned in a grid-like shape (cf. Figure 5). These scenarios were selected to investigate the influence of imbalanced distribution of neighbors in the communication area. In addition, different values for the communication radius $r$ were tested to investigate the influence of the neighborhood size which was identified to be a potential source of error (cf. Section 2.1).

\subsection{Distance Estimation between Neighbors}

In the first set of experiments, every node estimates its distance to all adjacent nodes using the GeoDE approach. For comparison, the average distance between adjacent nodes in the considered scenarios is taken as reference. To evaluate the quality of the estimates, the mean absolute percentage error (MAPE) is 


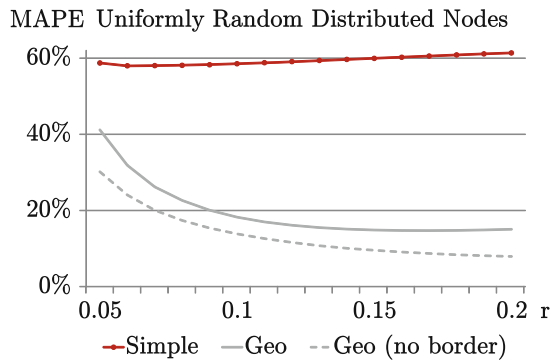

(a) Scenario 1

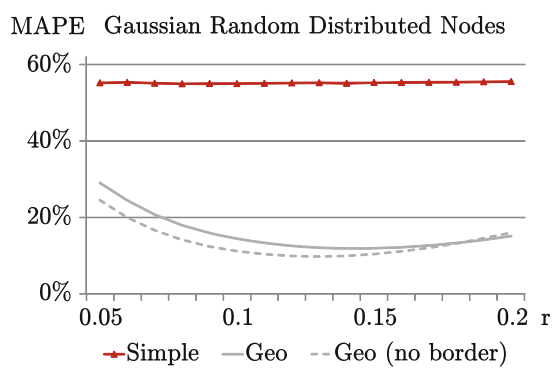

(b) Scenario 2

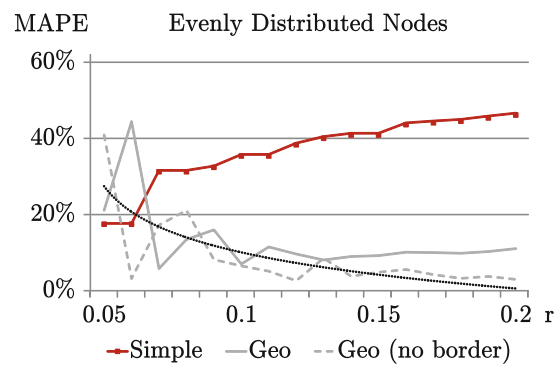

(c) Scenario 3

Fig. 6. MAPE using the geometric approach (Geo) compared to the error when using the average distance as an estimate (Simple)

calculated as $\operatorname{MAPE}\left(\hat{d}_{i j}\right)=\frac{\left|d_{i j}-\hat{d}_{i j}\right|}{d_{i j}}$, where $d_{i j}$ denotes the euclidean distance between a node $i$ and its neighbor $j$ and $\hat{d}_{i j}$ denotes the estimate of that distance. The MAPE gives information about the relative deviation of the estimate with respect to the real distance. As nodes near the border of the environment have a cropped communication area, all experiments were repeated using only inner nodes in order to illustrate the influence of border nodes on the network's average estimation error.

The results for Scenario 1 are shown in Figure 6(a). The GeoDE delivers estimation results ranging between $40 \%$ up to approximately $15 \%$ (10\% for inner nodes) deviation from the real distance which is consistently less error-prone than estimating the distance using the average of the network. The results indicate that the GeoDE approach delivers reliable estimates for distances between adjacent nodes. Furthermore, the quality of the estimation improves with increasing communication radius $r$. This can be explained by the entailed growth of the number of neighbors.

Figure 6(b) shows the MAPE for distance estimation between any two adjacent nodes in a Gaussian random distributed network. In contrast to what one 
might intuitively expect, the geometrical estimation performs even better as in uniformly random distributed networks despite the imbalanced distribution of nodes. The reason lies in averaging the estimates of both involved nodes. An unbalanced distribution of nodes leads to an overestimation in one node and an underestimation in the other node which may, under certain circumstances, provide a good estimate on average. Another factor for the less error-prone estimates in the Gaussian distributed network is the larger average neighborhood size due to the concentration of nodes in the center of the environment.

For scenario 2, it is further noticeable, that the percentage error does not decrease continuously with rising radius $r$, which seemed to be the case for uniformly random distributed networks. Instead, the curve has a convex shape. This is due to the approximation error of $f$. As stated before, the estimation error induced by approximating the function $f$ depends on $\theta$, i.e. the ratio of height $h$ to radius $r$. For all experiments $\theta$ ranges between $(0.61,0.69)$, thus the closest zero-error point $\theta *$ lies approximately at $\theta *=0.745$ (cf. Figure 4). Figure 7] shows the average percentage deviation for all considered node distributions and radii from this zero-error-point. The experiments with Gaussian distributed nodes diverge stronger with increasing radius than the experiments with uniformly random distributed nodes, which explains the convex behavior of the MAPE curve.

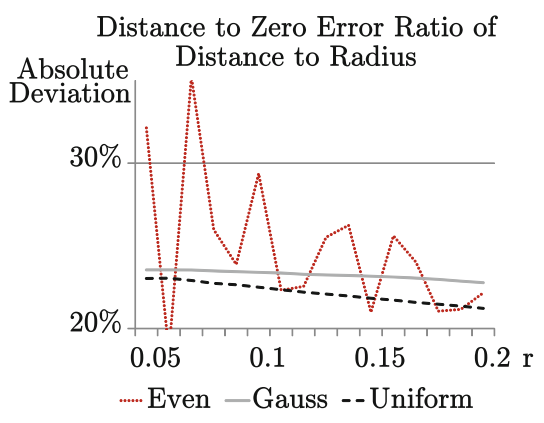

Fig. 7. Percentage deviation between $\theta$ and $\theta *$

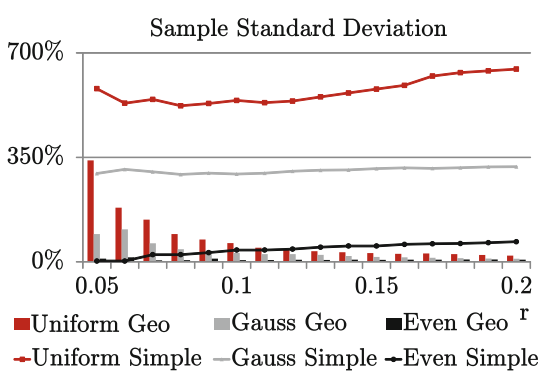

Fig. 8. Sample standard deviation for GeoDE between neighbors

Figure 6(c) shows the results for Scenario 3. Intuitively one would expect a similar MAPE as in uniformly random distributed networks, as the distribution of nodes is very balanced in both scenarios. Nevertheless, this does not appear to be the case at first sight, but when looking at the trendline (black dotted line) the behavior is quite similar. The oscillating error can be explained by the step-like increase of the average distance $d$ due to the symmetric arrangement (cf. Figure 9) in combination with the afore mentioned distance dependent error of the approximated function $f$.

Figure 8 illustrates the sample standard deviation for the previously presented experiments. It shows that the standard deviation is relatively small compared 


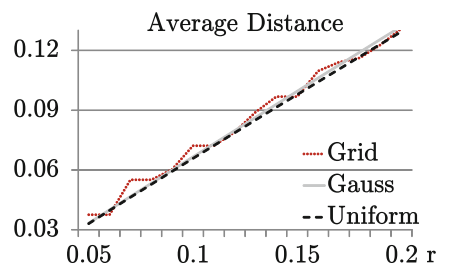

Fig. 9. Average distances between adjacent nodes in networks with different distributions depending on the communication radius $r$

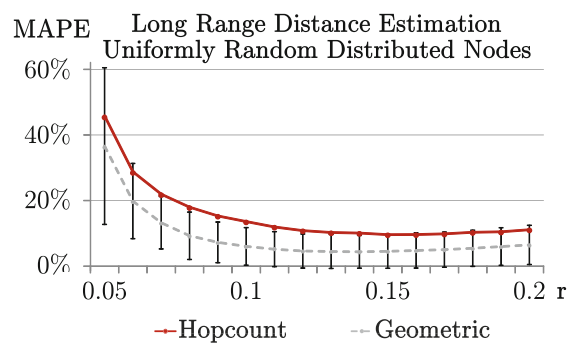

(a) Scenario 1

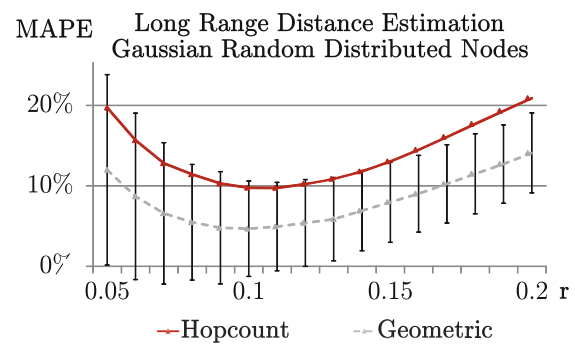

(b) Scenario 2

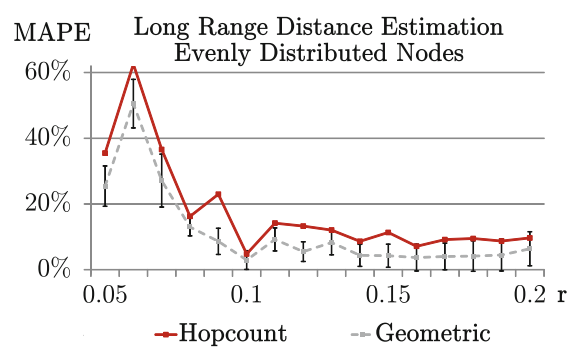

(c) Scenario 3

Fig. 10. MAPE for geometric versus traditional approach on long distance estimation including standard sample deviation

to the estimates using the average distance. This further substantiates the observation that the geometric concept is successfully transferred to the network topology delivering reliable estimates for each regarded distance estimation and not only on average for the whole network.

\subsection{Distance Estimation to Anchor Nodes}

The second set of experiments has the objective to evaluate the GeoDE concept for the estimation of distances to anchor nodes. Therefore, an anchor node is randomly chosen in each experiment iteration and all other nodes estimate their 
distance to this anchor node according to Algorithm 2 (cf. Section[2.2). For comparison, the hop count based distance estimation described in 29] is used. This method has been successfully used for localization in [15] and does not require more than one anchor node for distance estimation as opposed to the DV-hop propagation model in [27].

Figure 10(a) shows the MAPE for Scenario 1, using the uniform random distribution for node positioning. Figure 10(b) for Scenario 2, the Gaussian randomly distributed network and Figure 10(c) for Scenario 3, with evenly distributed nodes. It can be observed that the GeoDE approach leads to less error-prone estimates than the hop count based estimation for all considered distributions and radii. Furthermore, it should be noted that even the sample standard deviation is much less or equal to the MAPE of hop count based estimates. This confirms that the GeoDE approach is a consistent improvement in distance estimation for all considered ad hoc network scenarios and radii.

\section{Conclusion and Future Work}

This paper presents a new approach for a distributed distance estimation in an ad hoc network. The method relies on the ratio of shared to total neighbors and applies geometric coherences to the network structure. Three sources for error in the GeoDE approach were identified and, where possible, quantified. Experiments were conducted to investigate the absolute percentage error of the distance estimates in three different network scenarios: uniformly random, Gaussian random, and evenly distributed nodes. The results were compared to a hop count based estimation approach, showing that the GeoDE reliably delivers more precise estimates. This observation was consistent for all investigated communication radii and node distribution scenarios. Furthermore, even the sample standard deviation for GeoDE is close to the average percentage error of the hop count based approach and lies below it for some considered experiment settings. In future work, the GeoDE method is to be investigated for the usage in localization algorithms. We expect to improve the accuracy of the established coordinate system with the GeoDE as a great part of the error in finding coordinates is due to inaccuracy in distance estimation. Besides, the robustness of the algorithm is to be tested under mobile conditions.

\section{References}

1. Szewczyk, R., Osterweil, E., Polastre, J., Hamilton, M., Mainwaring, A., Estrin, D.: Habitat monitoring with sensor networks. Communications of the ACM 47(6), 34-40 (2004)

2. Werner-Allen, G., Lorincz, K., Johnson, J., Lees, J., Welsh, M.: Fidelity and yield in a volcano monitoring sensor network. In: Proceedings of the 7th Symposium on Operating Systems Design and Implementation (OSDI 2006), pp. 381-396. USENIX Association (2006) 
3. Cui, J.H., Kong, J., Gerla, M., Zhou, S.: The challenges of building mobile underwater wireless networks for aquatic applications. IEEE Network 20(3), 12-18 (2006)

4. Maihofer, C.: A survey of geocast routing protocols. IEEE Communications Surveys Tutorials 6(2), 32-42 (2004)

5. Li, J., Jannotti, J., De Couto, D.S.J., Karger, D.R., Morris, R.: A scalable location service for geographic ad hoc routing. In: Proceedings of the 6th Annual International Conference on Mobile Computing and Networking (MobiCom 2000), pp. 120-130. ACM (2000)

6. Amouris, K.N., Papavassiliou, S., Li, M.: A position-based multi-zone routing protocol for wide area mobile ad-hoc networks. In: Proceedings of the 49th IEEE Conference on Vehicular Technology, vol. 2, pp. 1365-1369. IEEE (1999)

7. Navas, J.C., Imielinski, T.: GeoCast - Geographic Addressing and Routing. In: Proceedings of the 3rd Annual ACM/IEEE International Conference on Mobile Computing and Networking MobiCom 1997, pp. 66-76. ACM (1997)

8. Liao, W., Tseng, Y., Sheu, J.: GRID: a fully location-aware routing protocol for mobile ad hoc networks. Telecommunication Systems 18(1-3), 37-60 (2001)

9. Gehrke, J., Madden, S.: Query processing in sensor networks. IEEE Pervasive Computing 3(1), 46-55 (2004)

10. Coore, D.: Botanical Computing: A Developmental Approach to Generating Interconnect Topologies on an Amorphous Computer. PhD thesis, MIT Department of Electrical Engineering and Computer Science (1999)

11. Nagpal, R.: Programmable Self-Assembly: Constructing Global Shape using Biologically-inspired Local Interactions and Origami Mathematics. PhD thesis, MIT Department of Electrical Engineering and Computer Science (2001)

12. Bachrach, J., Taylor, C.: Localization in sensor networks. In: Handbook of Sensor Networks, pp. 277-310. John Wiley \& Sons, Inc. (2005)

13. Allen, M., Baydere, S., Gaura, E., Kucuk, G.: Evaluation of localization algorithms. In: Mao, G., Fidan, B. (eds.) Localization Algorithms and Strategies for Wireless Sensor Networks. IGI Global (2009)

14. Savarese, C., Rabaey, J.M., Langendoen, K.: Robust positioning algorithms for distributed Ad-Hoc wireless sensor networks. In: Proceedings of the General Track of the Annual Conference on USENIX Annual Technical Conference, pp. 317-327. USENIX Association (2002)

15. Nagpal, R., Shrobe, H., Bachrach, J.: Organizing a Global Coordinate System from Local Information on an Ad Hoc Sensor Network. In: Zhao, F., Guibas, L.J. (eds.) IPSN 2003. LNCS, vol. 2634, pp. 333-348. Springer, Heidelberg (2003)

16. Coore, D.: Establishing a coordinate system on an amorphous computer. MIT/LCS/TR MIT/LCS/TR-737 (1998)

17. Bulusu, N., Heidemann, J., Estrin, D.: GPS-less low-cost outdoor localization for very small devices. IEEE Personal Communications 7(5), 28-34 (2000)

18. Bulusu, N., Bychkovskiy, V., Estrin, D., Heidemann, J.: Scalable, ad hoc deployable, rf-based localization. In: Proceedings of the Grace Hopper Celebration of Women in Computing. Institute for Women and Technology (2002)

19. Simic, S., Sastry, S.S.: Distributed localization in wireless ad hoc networks. Technical Report UCB/ERL M02/26, EECS Department, University of California, Berkeley (2002)

20. Savvides, A., Han, C.C., Strivastava, M.B.: Dynamic fine-grained localization in Ad-hoc networks of sensors. In: Proceedings of the 7th Annual International Conference on Mobile Computing and Networking (MobiCom 2001), pp. 166-179. ACM (2001) 
21. Niculescu, D., Nath, B.: Ad hoc positioning system (APS). In: Proceedings of the IEEE Global Telecommunications Conference, GLOBECOM 2001, vol. 5, pp. 2926-2931. IEEE (2001)

22. Hightower, J., Borriello, G., Want, R.: SpotON: an indoor 3D location sensing technology based on RF signal strength. Techreport 2000-02-02, University of Washington (2000)

23. Rappaport, T.S.: Wireless Communications: Principles and Practice, 2nd edn. Prentice-Hall (2002)

24. Bahl, P., Padmanabhan, V.N.: RADAR: an in-building RF-based user location and tracking system. In: Proceedings of the 19th Annual Joint Conference of the IEEE Computer and Communications Societies (IEEE INFOCOM 2000), pp. 775-784. IEEE (2000)

25. Werb, J., Lanzl, C.: Designing a positioning system for finding things and people indoors. IEEE Spectrum 35(9), 71-78 (1998)

26. Priyantha, N.B., Chakraborty, A., Balakrishnan, H.: The cricket location-support system. In: Proceedings of the 6th Annual International Conference on Mobile Computing and Networking (MobiCom 2000), pp. 32-43. ACM (2000)

27. Niculescu, D., Nath, B.: Dv based positioning in ad hoc networks. Telecommunication Systems 22, 267-280 (2003)

28. Savvides, A., Park, H., Srivastava, M.B.: The n-hop multilateration primitive for node localization problems. Mobile Networks and Applications 8(4), 443-451 (2003)

29. Nagpal, R.: Organizing a global coordinate system from local information on an amorphous computer. MIT A.I. Laboratory (A.I. Memo No. 1666, MIT) (1999)

30. Wong, S.Y., Lim, J.G., Rao, S.V., Seah, W.K.G.: Density-aware hop-count localization (DHL) in wireless sensor networks with variable density. In: Proceedings of 2005 IEEE Wireless Communications and Networking Conference, vol. 3, pp. 1848-1853. IEEE (2005)

31. Liu, Q., Pruteanu, A., Dulman, S.: GDE: a distributed gradient-based algorithm for distance estimation in large-scale networks. In: Proceedings of the 14th ACM International Conference on Modeling, Analysis and Simulation of Wireless and Mobile Systems, MSWiM 2011, pp. 151-158. ACM Press (2011)

32. Buschmann, C., Hellbrück, H., Fischer, S., Kröller, A., Fekete, S.P.: Radio Propagation-Aware Distance Estimation Based on Neighborhood Comparison. In: Langendoen, K.G., Voigt, T. (eds.) EWSN 2007. LNCS, vol. 4373, pp. 325-340. Springer, Heidelberg (2007)

33. Villafuerte, F.L., Terfloth, K., Schiller, J.: Using network density as a new parameter to estimate distance. In: Proceedings of the 7th International Conference on Networking, ICN 2008, pp. 30-35. IEEE Computer Society (2008)

34. Huang, B., Yu, C., Anderson, B., Mao, G.: Connectivity-Based distance estimation in wireless sensor networks. In: Proceedings of the 2010 Global Telecommunications Conference, pp. 1-5. IEEE Computer Society (2010) 